\title{
Macroglobulinemia and autoinflammatory disease
}

\author{
Brianne Navetta-Modrov, Qingping Yao*
}

Division of Rheumatology, Allergy and Immunology, Stony Brook University Renaissance School of Medicine, Stony Brook, NY, USA

Abstract

Received August 04, 2021 accepted August 19, 2021

Macroglobulinemia is associated with Schnitzler syndrome (SchS) and Waldenstrom macroglobulinemia (WM). The aim of this article was to review the above-mentioned two diseases from clinical aspects and their potential genetic links. We performed a PubMed search using the following keywords: "SchS," "WM," "autoinflammatory disease," "periodic fever syndrome," and "nucleotide-binding oligomerization domain containing protein 2 (NOD2)." A case is exemplified. Both SchS and WM share some clinical phenotypes, and SchS can evolve into WM. Though no genetic link to SchS has been established, myeloid differentiation primary response gene 88 (MyD88) mutations are detected in one-third of SchS patients and $86 \%$ WM patients. Genetic analysis of periodic fever syndrome genes has detected NOD2 mutations in $18 \%$ SchS patients and rarely NLRP3 mutations. The literature data suggest that both MyD88 and NOD2 mutations may contribute to SchS. Both MyD88 and NOD2 are known to play important roles in innate immune response, and they may be cooperative in certain autoinflammatory diseases. Molecular analysis of NOD2 mutations may be incorporated into genetic testing for patients with suspected SchS or SchS/WM.

Keywords

autoinflammatory disease • macroglobulinemia $・$ MyD88 $\cdot$ NOD2 $\cdot$ Schnitzler syndrome $\cdot$ Waldenstrom macroglobulinemia

\section{Introduction}

Macroglobulinemia refers to elevated immunoglobulin $\mathrm{M}$ in serum and is associated with diseases such as Waldenstrom macroglobulinemia (WM) ${ }^{[1]}$ and Schnitzler syndrome (SchS). ${ }^{[2]}$ WM is one of the lymphoproliferative disorders. SchS is a rare autoinflammatory disease, and specific genetic mutations have not been identified yet. To our knowledge, macroglobulinemia has not been reported in other autoinflammatory disease or periodic fever syndromes. In this article, we present a case of WM with molecular evidence of combined gene mutations for autoinflamamtory diseases. In conjunction with the literature, hypergammaglobulinemia in association with autoinflammatory disease is reviewed and discussed.

\section{Methods}

We performed a PubMed search using the following keywords: "SchS," "WM," "autoinflammatory disease," "periodic fever syndrome" and "nucleotide-binding oligomerization domain containing protein 2 (NOD2)." A case is exemplified. Two diseases, WM and SchS, were summarized from clinical and molecular aspects.

\section{Results}

\section{Case Report}

A 51-year-old Caucasian female with WM presented for evaluation of chronic cough and recurrent rash. The patient had recurrent infections since childhood, including chronic bronchitis/asthma, multiple pneumonias, and one prolonged influenza infection. She was a former smoker. The patient was diagnosed with WM 5 years ago when she had recurrent cervical lymphadenopathy. Meanwhile, she also began having recurrent urticarial lesions described as erythematous patches on her neck and back, which she initially thought was allergy to polyester. Her rash was intensely pruritic lasting less than $24 \mathrm{~h}$ in one location. Chronic urticaria was diagnosed by an allergist without a skin biopsy, and the rash responded

\footnotetext{
Address for correspondence:

*Qingping Yao, MD, PhD, Division of Rheumatology, Allergy and Immunology,

Stony Brook University Renaissance School of Medicine, Stony Brook, NY

11794, USA. E-mail: qingping.yao@stonybrookmedicine.edu
} 
well to cetirizine $10 \mathrm{mg}$ daily. The patient had bouts of mild nonbloody diarrhea without abdominal pain, and she did not have a colonoscopy. She had arthralgia with recurrent bilateral lower leg swelling from the knees down to the feet over the last 20 years. She denied recurrent fevers, sore throat, ear infections, and myalgia. Her half-sister had WM without a family history of periodic fever syndromes. Physical examination was unremarkable under our care.

Hematological workup showed normal CBC. Serum protein electrophoresis revealed a restricted band (M-spike) and serum immunofixation noted IgM-Lambda monoclonal protein, with a low free kappa/lambda ratio of $0.19(0.26-1.65)$. A bone marrow biopsy revealed a nodular and interstitial lymphoplasmacytic infiltrate comprising approximately $25-30 \%$ of the total cellularity with evidence of lambda light chain restriction. CD20 staining identified approximately $25 \%$ B cell infiltrate, while CD138 highlighted the plasmacytic component of the neoplastic infiltrate to be approximately $5 \%$. There was few kappa-positive cells and evidence of lambda light chain restriction. Mild diffuse reticulin fibrosis was also noted. Flow cytometry analysis of bone marrow aspirates confirmed the presence of lambda light chain restricted B-cell (4\%) and plasma cell $(1 \%)$ populations. Furthermore, myeloid differentiation primary response gene 88 (MYD88) mutation p.L265P was identified. Serum lgM level was $2626 \mathrm{mg} / \mathrm{dL}(48-271 \mathrm{mg} / \mathrm{dL})$, while the levels of $\lg G$, IgA, and IgE were slightly low. WM was diagnosed by an oncologist and conservatively treated without any chemotherapy. A Pan CT scan reported mild emphysema with tree-in-bud nodularity at the peripheral left lung base compatible with infectious/inflammatory bronchiolitis, and right middle lobe linear atelectasis/scarring. Her condition was stable.

Given her presentation, such as rash, arthralgia, lower leg swelling, and diarrhea, as well as elevated ESR, an autoinflammatory disease was suspected, and a six gene periodic fever syndrome panel was completed. She was found to have the following heterozygous variants: TNFRSF1A c.362G>T, p. Arg121Gln (R92Q), NOD2 Intron 8 c. $2798+158 C>T$ (IVS8 + 158) and Exon 11 c.3019dupC (1007fs).

\section{Waldenstrom Macroglobulinemia (WM)}

WM is a rare low grade B cell lymphoma characterized by infiltration of lgM-secreting lymphoplasmacytic cells in the bone marrow and other organs. The disease was initially described by Jan G. Waldenstrom, a Swedish physician in 1944 and is associated with IgM monoclonal gammopathy. ${ }^{[3]}$

\section{Clinical Manifestation and Diagnosis}

Clinical features of WM include IgM paraprotein, lymphadenopathy, splenomegaly, monoclonal gammopathy of unknown significance, autoimmune phenomena, increased lactate dehydrogenase level, amyloidosis, cryoglobulinemia, hyperviscosity syndrome, large cell transformation, extranodal disease, and chronic renal failure. Asymptomatic disease at diagnosis accounted for $33 \% .{ }^{[4]} \mathrm{WM}$-associated rash is extremely rare. Reports of rash occurring in WM patients are mostly from SchS evolving into WM and chemotherapy related. ${ }^{[5,6]}$

Criteria incorporating clinical, morphologic, and immunophenotypic parameters have been proposed for an accurate diagnosis of WM. Diagnostic criteria were: IgM monoclonal gammopathy of any concentration, bone marrow infiltration by small lymphocytes, plasmacytoid cells, and plasma cells in a diffuse, interstitial or nodular pattern, and a surface $\lg (+)$ CD19(+)CD20(+)CD5(-)CD10(-)CD23(-) immunophenotype. [7]

\section{MyD88}

MYD88 was first described in $1990 .{ }^{[8]}$ MyD88 is a cytoplasmic adaptor protein that is a central player in innate immune signaling. ${ }^{[9]}$ MyD88 mediates interaction of toll-like receptors (TLR) and IL-1 receptor families and their downstream kinases, leading to activation of NF-kB and mitogen-activated protein kinases (MAPK) pathways. MyD88 deficient mice have been used as a model for TLR deficiency being susceptible to a variety of bacterial pathogens and parasites. ${ }^{[10]}$

MyD88 L265P was identified in 2012 and is a commonly recurrent mutation in patients with WM. This mutation can be useful in differentiating WM and non-IgM lymphoplasmacytic lymphoma from B-cell disorders that have some of the same features. ${ }^{[1]}$ MYD88 L265P mutation is present in 86\% WM patients ${ }^{[12]}$ and is specifically associated with $\mathrm{WM}$ and IgM monoclonal gammopathy of unknown significance. It is also seen in splenic marginal zone lymphoma, IgM amyloidosis, and mucosa-associated lymphatic tissue lymphoma. ${ }^{[1]}$ It is reported that WM patients with MyD88 wild type genotype have a shorter time transformation to more aggressive histology compared to Myd88 L265P genotype. The histological transformation confers an inferior overall survival in patients with WM. ${ }^{[13]}$

\section{Schnitzler Syndrome (SchS)}

SchS is characterized by urticaria, fever, musculoskeletal pain, lymphadenopathy, hepatosplenomegaly, and monoclonal gammopathy. SchS was first reported by Dr. Liliane Schnitzler, a French dermatologist in 1972. ${ }^{[14]}$

\section{Clinical manifestation and diagnosis ${ }^{[2,15]}$}

Based on Lipsker criteria, SchS can be diagnosed if two major and two minor criteria are met. Based on the Strasbourg 
Table 1. Diagnostic criteria for SchS

\begin{tabular}{lll}
\hline Criteria & Lipsker Criteria & Strasbourg Criteria \\
\hline Major & Urticarial rash & Chronic urticarial rash \\
& Monoclonal lgM component & Monoclonal lgM or IgG \\
& Recurrent fever \\
& Fever & Objective findings of abnormal bone remodeling \\
& Arthralgia/arthritis & with or without bone pain \\
& Bone pain & Neutrophilic dermal infiltrate \\
Minor & Palpable lymph node & Leukocytosis and/or elevated CRP \\
& Liver or spleen enlargement & \\
& Elevated ESR & \\
& Leukocytosis & \\
& Abnormal findings on bone morphologic investigation & \\
\hline
\end{tabular}

criteria, a definite SchS can be diagnosed if two major (obligate) and two minor criteria are fulfilled. Probable diagnosis can be made if two obligate and one minor criterion are identified. Both Lipsker and Strasbourg criteria are based on clinical manifestations and routine laboratory findings, and in the latter criteria dermopathology was included. But there is a lack of molecular or genetic makers in both criteria.

\section{Therapy for SchS}

Conventional drugs such as nonsteroidal anti-inflammatory drugs, antihistamines, and glucocorticoids may be tried. ${ }^{[2]}$ Colchicine was anecdotally effective for the disease. ${ }^{[16]}$ Both anecdotal reports and clinical trials have shown effectiveness of interleukin-1 inhibitors for the treatment of the disease. In a phase two clinical trial, Canakinumab is effective over 4-year-period observation with mild adverse reactions. ${ }^{[17,18]}$ Another prior clinical trial has also proven the efficacy of Canakinumab. ${ }^{[19]}$ In an open label study, Rilonacept is effective and well-tolerated in patients with SchS. ${ }^{[20]}$ Anakinra was effective in an anecdotal report, ${ }^{[21]}$ but no clinical trial of the biologic was seen.

IL-6 antagonist was effective in anecdotal case reports. ${ }^{\text {[22, 23] }}$ In a recent open label study, ${ }^{[24]}$ eight SchS patients received tocilizumab for 20 weeks. Tocilizumab treatment reduced clinical symptoms and inflammatory markers in SchS. However, loss of drug efficacy was observed in most patients over time.

\section{Discussion}

\section{Association between SchS and WM}

SchS may evolve into WM. For example, in a study of nine patients with SchS, four patients progressed to lymphoproliferative disorder, WM. ${ }^{[5]}$ One study has found that approximately $15-20 \%$ of SchS patients would eventually develop a lymphoproliferative disorder like WM. ${ }^{[25]}$ Therefore, a hypothesis of shared pathophysiological mechanism between these two diseases has been proposed. ${ }^{[26]}$

What could be the mechanisms behind the association between SchS and WM? NLRP3 inflammasome is involved in SchS patients, and a therapy with IL-1 inhibitors is effective for the disease. ${ }^{[20]}$ NLRP3 inflammasome plays a role in B cell secretion of IgM and adaptive $B$ lymphocyte response by modulating production of IgM and IL-1b in SchS. ${ }^{[27]}$ In a study of $B$ cell VDJ recombination and properties of the resulting immunoglobulin chains using next-generation sequencing, specific B cell expansions have been found in SchS patients. ${ }^{[28]}$

Interestingly, MyD88 mutations have been shared by both SchS and WM. In a study of 30 patients with SchS, one third of SchS patients (9/30) harbored MyD88 mutations. ${ }^{[29]}$ In addition, there has been a case report of an association of MyD88 L265P mutation with SchS. ${ }^{[30]}$ These data suggest that both NLRP3 inflammasome and MyD88 may contribute to the association of these two diseases.

\section{Periodic Fever Syndrome Gene Mutations in SchS Patients}

SchS has no established genetic association to date. Besides MyD88 mutations, gene mutations for periodic fever syndromes were examined in SchS patients. Our literature search has identified one study of molecular analysis of periodic fever genes in SchS patients. In this study, genetic analyses of 32 gene panels associated with inherited autoinflammatory diseases were conducted in 21 SchS patients. The study used Sanger/next generation sequencing (NGS) and identified NLRP3 V198M in one patient and NOD2 variants in 3 patients (R684W in one, p.M863V and p.A1006fs in one and T189M in the other). ${ }^{[11]}$ We would believe that these 3 reported cases of SchS with NOD2 mutations could reasonably be diagnosed with Yao syndrome (YAOS, OMIM 617321) rather than variant-type SchS if these patients did not have neutrophilic urticarial dermatosis. Despite anecdotal report 
of NLRP3 V198M identified in a SchS patient, ${ }^{[32]}$ Pathak J, et al. reported no NLRP3 mutations identified in 30 patients with SchS. ${ }^{[29]}$

Our patient had WM and also presented with intermittent rash described as erythematous patches, arthralgia/lower leg swelling, and diarrhea. The phenotype and presence of the compound NOD2 variants IVS8+158 and 1007fs is diagnostic of YAOS, formerly called NOD2-associated autoinflammatory disease. YAOS is characterized by recurrent fever, dermatitis, arthralgia, distal lower extremity swelling, gastrointestinal and sicca symptoms with eyelid swelling, and this disease is associated with specific NOD2 mutations. ${ }^{[33]}$ The diagnostic criteria proposed for the disease include the characteristic phenotype and NOD2 genotype, as well as exclusion of relevant diseases as specified in the diagnostic criteria. ${ }^{[34]}$ YAOS patients typically present with erythematous patches consistent with spongiotic dermatitis on a skin biopsy. The presence of urticaria-like rash and monoclonal IgM in our case is also reminiscent of possible SchS that typically has chronic urticarial rash with neutrophilic infiltration on a skin biopsy, i.e., neutrophilic urticarial dermatosis. ${ }^{[35]}$ Our case as well as the literature data suggests that NOD2 mutations may contribute to SchS-like clinical manifestations, and NOD2 whole gene sequencing may be integrated into periodic fever syndrome gene panel in suspected patients with SchS or WM.

To our knowledge, there have been no reports of IgM elevation in association with TNF receptor associated periodic syndrome (TRAPS) in the literature. In our case, TNFRSF1A $\mathrm{R} 92 \mathrm{Q}$, a low penetrance variant, was identified. However, the patient did not have a characteristic phenotype to support TRAPS. The significance of this variant is unclear in SchS and WM, and it could be coincidental or noncontributory in this case.

\section{NOD2 and Humoral Immune Response}

Given the identification of both NOD2 and MyD88 gene mutations in some SchS and WM patients, we would assume that these two gene mutations or molecules might contribute to the shared pathophysiology of both SchS and WM.
NOD2 was first reported in 2001 as a cytosolic protein. Upon recognition of muramyl dipeptides (breakdown products of bacterial peptidoglycans), ${ }^{[36]}$ NOD2 functions through RICK or RIP2 to activate p38 MAPK and NF-kB, resulting in inflammatory response, and enhanced autophagic activity. ${ }^{[37]}$ NOD2 mutations are associated with autoinflammatory diseases' such as Crohn's disease, Blau syndrome, and YAOS. The relationship between humoral immune response and NOD2 has been studied. In a study of modulation of adaptive immunity by different adjuvant-antigen combinations in mice lacking NOD2, NOD2 was required for an optimal IgG1 and IgG2c response in the absence of exogenous TLR or NLR agonists. ${ }^{[38]}$

\section{Relationship between MyD88 and NOD2}

Our patient carries both MYD88 L265P.and NOD2 mutations, thus raising a question whether both molecules are cooperative. MyD88 in B cells is essential for antibody production when antigens are co-administered with TLR agonists. ${ }^{[39]}$ In the literature, a potential relationship between NOD2 and MyD88 has been studied. ${ }^{[40]}$ Most of such studies were conducted in infections, particularly in mouse models of infections such as Staphaureus ${ }^{[41]}$ and Turberculosis. ${ }^{[42]}$ Very few studies were based on autoinflammatory disease. In a study of mouse model, ocular inflammation induced by synthetic TLR2 agonists required MyD88 but not NOD2 or NOD1. ${ }^{[43]}$ In a mouse model of peptidoglycan induced arthritis, TLR-2 or MyD88 deficiency did not influence arthritis induced by specific NOD2 agonist. ${ }^{[44]}$ However, in a study of NOD2 and MyD88 double deficient mice, there was a significant reduction in the innate immune response compared to mice deficient with either one of two genes alone, suggesting that NOD2 and TLR/MyD88 pathways would cooperate independently but additively to stimulate innate immune response. ${ }^{[45]}$ Taken together, NOD2 and MyD88 pathways could be cooperative in certain diseases under circumstances.

In summary, macroglobulinemia can occur in autoinflammatory diseases. SchS patients may be complicated with WM. Both MyD88 and NOD2 mutations can be detected in some SchS patients, and they could be cooperative in contributing to SchS that evolves into WM.

Conflict of interest

Qingping Yao is an Editorial Board Member of the journal. The article was subject to the journal's standard procedures, with peer review handled independently of this member and his research groups.

Funding

The authors categorically state that there is no financial funding for this study. 
References

[1] Gertz MA. Waldenstrom Macroglobulinemia: 2021 Update on Diagnosis, Risk Stratification, and Management. Am J Hematol. 2021;96(2):258-269.

[2] Gusdorf L, Lipsker D. Schnitzler Syndrome: A Review. Curr Rheumatol Rep. 2017;19(8):46.

[3] Kaseb H, Gonzalez-Mosquera LF, Parsi M, et al. Lymphoplasmacytic Lymphoma. Treasure Island, FL. StatPearls, 2021.

[4] Owen RG, Barrans SL, Richards SJ, et al. Waldenstrom macroglobulinemia. Development of Diagnostic Criteria and Identification of Prognostic Factors. Am J Clin Pathol. 2001;116(3):420-428.

[5] Gameiro A, Gouveia M, Pereira M, et al. Clinical Characterization and Long-Term Follow-Up of Schnitzler Syndrome. Clin Exp Dermatol. 2016;41(5):461-467.

[6] Jensen AB, Stausbol-Gron B, Riber-Hansen R, et al. IbrutinibAssociated Skin Toxicity: A Case of Maculopapular Rash in a 79-Year Old Caucasian Male Patient with Relapsed Waldenstrom's Macroglobulinemia and Review of the Literature. Dermatol Rep. 2017;9(1):6976. [7] Owen RG. Developing diagnostic criteria in Waldenstrom's macroglobulinemia. Semin Oncol. 2003;30(2):196-200.

[8] Lord KA, Hoffman-Liebermann B, Liebermann DA. Nucleotide Sequence and Expression of a cDNA Encoding MyD88, A Novel Myeloid Differentiation Primary Response Gene Induced by IL6. Oncogene. 1990;5(7):1095-1097.

[9] Dishon S, Schumacher A, Fanous J, et al. Development of a novel backbone cyclic peptide inhibitor of the innate immune TLR/IL1R signaling protein MyD88. Sci Rep. 2018;8(1):9476.

[10] Deguine J, Barton GM. MyD88: A Central Player in Innate Immune Signaling. F1000prime Rep. 2014;6:97.

[11] Treon SP, Xu L, Yang G, et al. MYD88 L265P Somatic Mutation in Waldenstrom's Macroglobulinemia. N Engl J Med. 2012;367(9): 826-833.

[12] Jimenez C, Sebastian E, Chillon MC, et al. MYD88 L265P is A Marker Highly Characteristic of, but not Restricted to, Waldenstrom's Macroglobulinemia. Leukemia. 2013;27(8):1722-1728.

[13] Zanwar S, Abeykoon JP, Durot E, et al. Impact of MYD88(L265P) Mutation Status on Histological Transformation of Waldenstrom Macroglobulinemia. Am J Hematol. 2020;95(3):274-281.

[14] Sokumbi O, Drage LA, Peters MS. Clinical and Histopathologic Review of Schnitzler Syndrome: The Mayo Clinic Experience (1972-2011). J Am Acad Dermatol. 2012;67(6):1289-1295.

[15] Lipsker D, Veran $Y$, Grunenberger F, et al. The Schnitzler Syndrome. Four New Cases and Review of the Literature. Medicine (Baltimore). 2001;80(1):37-44.

[16] Baresic M, Mitrovic J, Morovic Vergles J, et al. Different Therapeutic Paths (Colchicine vs. Anakinra) in Two Patients With Schnitzler's Syndrome. Arch Rheumatol. 2016; (4):377-380.

[17] Krause K, Bonnekoh H, Ellrich A, et al. Long-Term Efficacy of Canakinumab in the Treatment of Schnitzler Syndrome. J Allergy Clin Immunol. 2020;145(6):1681-1686.e5.

[18] Krause K, Tsianakas A, Wagner N, et al. Efficacy and Safety of Canakinumab in Schnitzler Syndrome: A Multicenter Randomized Placebo-Controlled Study. J Allergy Clin Immunol. 2017;139(4):
1311-1320.

[19] de Koning HD, Schalkwijk J, van der Ven-Jongekrijg J, et al. Sustained Efficacy of the Monoclonal Anti-Interleukin-1 Beta Antibody Canakinumab in a 9-month Trial in Schnitzler's Syndrome. Ann Rheum Dis. 2013;72(10):1634-1638.

[20] Krause K, Weller K, Stefaniak R, et al. Efficacy and Safety of the Interleukin-1 Antagonist Rilonacept in Schnitzler Syndrome: An Open-Label Study. Allergy. 2012;67(7):943-950.

[21] Sonnichsen A, Saulite I, Mangana J, et al. Interleukin-1 Receptor Antagonist (Anakinra) for Schnitzler Syndrome. J Dermatol Treat. 2016;27(5):436-438.

[22] Claus J, Vanderschueren S. Variable responses to tocilizumab in four patients with Schnitzler syndrome. J Clin Immunol. 2019;39(4):370-372.

[23] Yan R, Cao W, Liu X, et al. A Chinese Case Series of Schnitzler Syndrome and Complete Remission in one Tocilizumab-Treated Patient. Clin Rheumatol. 2020;39(12):3847-3852.

[24] Bonnekoh H, Frischbutter S, Roll S, et al. Tocilizumab Treatment in Patients with Schnitzler Syndrome: An Open-Label Study. J Allergy Clin Immunol Pract. 2021;9(6):2486-2489.e4.

[25] Simon A, van der Meer JW, Vesely R, et al. Approach to Genetic Analysis in the Diagnosis of Hereditary Autoinflammatory Syndromes. Rheumatology (Oxford). 2006;45(3):269-273.

[26] van Leersum FS, Potjewijd J, van Geel M, et al. Schnitzler's Syndrome - A Novel Hypothesis of A Shared Pathophysiologic Mechanism with Waldenstrom's Disease. Orphanet J Rare Dis. 2019;14(1):151.

[27] Ali MF, Dasari H, Van Keulen VP, et al. Canonical Stimulation of the NLRP3 Inflammasome by Fungal Antigens Links Innate and Adaptive B-Lymphocyte Responses by Modulating IL-1 beta and IgM Production. Front Immunol. 2017;8:1504.

[28] Pathak S, Rowczenio D, Lara-Reyna S, et al. Evidence of B Cell Clonality and Investigation Into Properties of the IgM in Patients With Schnitzler Syndrome. Front Immunol. 2020;11:569006.

[29] Pathak S, Rowczenio DM, Owen RG, et al. Exploratory Study of MYD88 L265P, Rare NLRP3 Variants, and Clonal Hematopoiesis Prevalence in Patients With Schnitzler Syndrome. Arthritis Rheumatol. 2019;71(12):2121-2125

[30] Goodman AM, Cohen PR, Li A, et al. Schnitzler Syndrome Associated with MYD88 L265P Mutation. JAAD Case Rep. 2019;5(4): 312-316.

[31] Rowczenio DM, Pathak S, Arostegui JI, et al. Molecular Genetic Investigation, Clinical Features, and Response to Treatment in 21 Patients with Schnitzler Syndrome. Blood. 2018;131(9):974-981.

[32] Loock J, Lamprecht P, Timmann C, et al. Genetic Predisposition (NLRP3 V198M Mutation) for IL-1-Mediated Inflammation in A Patient with Schnitzler Syndrome. J Allergy Clin Immunol. 2010;125(2):500-502.

[33] Yao Q, Su LC, Tomecki KJ, et al. Dermatitis as A Characteristic Phenotype of A New Autoinflammatory Disease Associated with NOD2 Mutations. J Am Acad Dermatol. 2013;68(4):624-631.

[34] Yao Q, Shen B. A Systematic Analysis of Treatment and Outcomes of NOD2-Associated Autoinflammatory Disease. Am J Med. 
2017;130(3):365.e13-365.e18.

[35] Gusdorf L, Lipsker D. Schnitzler Syndrome: the Paradigm of An Acquired Adult-Onset Auto-Inflammatory Disease. Giornale italiano di dermatologia e venereologia: organo ufficiale, Societa italiana di dermatologia e sifilografia 2020;155(5):567-573.

[36] Ogura Y, Inohara N, Benito A, et al. Nod2, a Nod1/Apaf-1 Family Member that is Restricted to Monocytes and Activates NF-KappaB. J Biol Chem. 2001;276(7):4812-4818.

[37] Yao Q. Nucleotide-Binding Oligomerization Domain Containing 2: Structure, Function, and Diseases. Semin Arthritis Rheum. 2013;43(1):125-130.

[38] Moreira LO, Smith AM, DeFreitas AA, et al. Modulation of Adaptive Immunity by Different Adjuvant-Antigen Combinations in Mice Lacking Nod2. Vaccine. 2008;26(46):5808-5813.

[39] Pasare C, Medzhitov R. Control of B-Cell Responses by Toll-Like Receptors. Nature. 2005;438(7066):364-368.

[40] Mitchell JA, Paul-Clark MJ, Clarke GW, et al. Critical Role of TollLike Receptors and Nucleotide Oligomerisation Domain in the Regu- lation of Health and Disease. J Endocrinol. 2007;193(3):323-330. [41] Sethi S, Chakraborty T. Role of TLR-/NLR-Signaling and the Associated Cytokines Involved in Recruitment of Neutrophils in Murine Models of Staphylococcus aureus Infection. Virulence. 2011;2(4):316-328. [42] Kleinnijenhuis J, Oosting M, Joosten LA, et al. Innate Immune Recognition of Mycobacterium tuberculosis. Clin Dev Immunol. 2011;2011:405310.

[43] Rosenzweig HL, Galster K, Vance EE, et al. NOD2 Deficiency Results in Increased Susceptibility to Peptidoglycan-Induced Uveitis in Mice. Invest Ophthalmol Vis Sci. 2011;52(7):4106-4112.

[44] Rosenzweig HL, Jann MJ, Vance EE, et al. Nucleotide-Binding Oligomerization Domain 2 and Toll-Like Receptor 2 Function Independently in A Murine Model of Arthritis Triggered by Intraarticular Peptidoglycan. Arthritis Rheum. 2010;62(4):1051-1059.

[45] Suzuki M, Cela R, Bertin TK, et al. NOD2 Signaling Contributes to the Innate Immune Response Against Helper-Dependent Adenovirus Vectors Independently of MyD88 in vivo. Hum Gene Ther. 2011;22(9):1071-1082. 